\title{
3 Ethische Herausforderungen im Krankenhaus
}

In diesem Kapitel werden die nach unserer Erfahrung wichtigsten ethischen Herausforderungen und Konflikte im Krankenhaus beschrieben. Wichtig ist dabei, dass diese Themen anderer Art sind als es nach der Lektüre akademischer Ethik-Literatur zu erwarten ist. An erster Stelle steht das Thema „Wertschätzung“ innerhalb der Mitarbeiterschaft einschließlich der Führungskräfte, das mehr ist als ein psychologisches oder kommunikatives Phänomen. Das von einer Klinik repräsentierte Menschenbild in Medizin und Pflege hat insofern einen „ethischen Kern“, als es die praktizierte Wertschätzung gegenüber den Patienten in allen Aspekten der Behandlung und Betreuung zum Ausdruck bringt. Therapieentscheidungen sind zweifellos ein zentrales ethisches Themenfeld, insbesondere dann, wenn es um Fragen des Lebenserhalts und des Therapiemaßes geht. Schließlich stellen die Konflikte zwischen medizinethischen Prinzipien und Unternehmensinteressen eine wachsende, auch auf gesellschaftlicher Ebene ungelöste ethische Herausforderung dar.

\subsection{Einleitung}

Ethische Herausforderungen im Krankenhaus können vielerlei Gestalt annehmen. Sie können sich um die Frage drehen, welche Art der Behandlung eines Patienten gewählt werden soll. Es kann darum gehen, wie grundlegende ethische Normen geschützt werden, sei es in der Patientenversorgung, im Personalmanagement, im Umgang miteinander oder im Umgang mit den Trägern, den Krankenkassen oder Zulieferern. Es kann 
sich um Ziele und Zielkonflikte des Managements handeln oder um den Charakter des Medizin- und Pflegekonzepts.

Für die Praxis ist es dabei nicht wichtig, ob „Ethik“ im Sinne akademischer Modelle korrekt bestimmt wird. Eher geht es um Antworten auf grundlegende Fragen zur Medizin, zur Patientenversorgung, zur Gestaltung des Klinikalltags, zur Organisation, zur Kultur. Dabei muss nicht immer explizit von Ethik die Rede sein. Viele Phänomene haben eine ethische Dimension, werden aber unter anderem Namen thematisiert. Dies gilt unter anderem für das Bild vom Patienten, das dem bevorzugten medizinischen Stil zugrunde liegt. Sieht man ihn eher als ein reparaturbedürftiges Objekt? Wird man seinem Person-sein gerecht? Werden spirituelle Phänomene berücksichtigt? Geht man achtsam miteinander um? Es kann nicht davon ausgegangen werden, dass alle Mitarbeiter eines Klinikums dasselbe verstehen, wenn von Ethik die Rede ist. Deshalb ist die konkrete Verständigung so wichtig. Mit ihr beginnt das Thema „Wertschätzung" genauso wie mit dem Grüßen, d.h. der Wahrnehmung und Achtung des Gegenübers.

\subsection{Wertschätzung als moralisches Phänomen und als ethische Herausforderung}

Eine ethische Herausforderung lässt sich im Kern daran erkennen, dass ein gefühlter oder empfundener Konflikt oder eine Kommunikation vorliegt, der oder die einen nicht kalt lässt, Emotionen freisetzt, Motivationen beeinflusst, Identifikationen fördert oder mindert, Zustimmung und Unterstützung oder Streit und Widerstand erzeugt. Erfolgreiche Führung setzt die Wahrnehmung moralischer Phänomene und ihre nachfolgende ethische Reflexion voraus, damit die Anvertrauten (,die Mitarbeiter“) sich mit ihren Aufgaben und Handlungen identifizieren können. Dies gelingt nur, wenn die Selbstachtung der Personen nicht verletzt wird. Von daher ist es verständlich, wenn in der Praxis ein Thema in den Vordergrund rückt, welches in der akademischen Ethik eher vernachlässigt wird: Wertschätzung.

„Eine Kommunikation nimmt moralische Qualität an, wenn und soweit sie menschliche Achtung oder Missachtung zum Ausdruck bringt.“ „Moral hat daher (...) eine Tendenz, Streit zu erzeugen oder aus Streit zu entstehen und den Streit dann zu verschärfen. Moral ist polemogener Natur. Wer immer bei Meinungsverschiedenheiten moralisch argumentiert, setzt seine Selbstachtung ein, um seinen Anforderungen und Argumenten Nachdruck zu verleihen. Es fällt dann schwer, den Rückzug anzutreten (...) So können Steppenbrände entstehen.“ (Luhmann 1989) 
Für klinische Ethik-Projekte, die oft mehrere Jahre lang durchgeführt werden, empfiehlt es sich, mit empirischen Methoden die vorhandene Kommunikationskultur und die aktuellen Konfliktfelder zu erfassen. Dies geschieht durch die Befragung von Mitarbeitern, durch zusätzliche offene Gesprächskreise, Mitarbeiterversammlungen, Vorträge und Diskussionsveranstaltungen zu ausgewählten Themen, durch Sprechstunden und durch teilnehmende Beobachtung an den Arbeitsabläufen. Hinzu kommt das Studium von Dokumenten, seien es Unternehmensgrundsätze, Patientenakten, Berichte des Qualitätsmanagements, Fehlermeldungen usw. Befragt man die Mitarbeiter danach, was sie sich von einem Ethik- Projekt erhoffen würden so muss davon ausgegangen werden, dass die wenigsten Mitarbeiter sich anfangs etwas unter einem „Ethik-Projekt“ vorstellen können und dass der wichtige Unterschied zwischen Ethik und Moral nicht bewusst ist. Wenn aber eine Erwartung immer wieder an erster Stelle genannt wird so heißt diese „mehr Wertschätzung“. Wertschätzung ist der Schlüssel zum Zugang der Führungskräfte zu ihren Mitarbeitern, die ein sehr feines Gespür dafür haben, ob diese „echt“ gemeint ist oder instrumentell eingesetzt wird.

\section{Wertschätzung ist der Schlüssel für den Zugang der Führungskräfte zu ihren Mitarbeitern.}

Gefühle mangelnder Wertschätzung entstehen, wenn man etwas „ist“, „gibt“ oder „erreicht“, dessen Wert von anderen nicht gewürdigt zu werden scheint. Diese Achtung erwarten zumeist Mitarbeiter von ihren Vorgesetzten. Aber auch Führungskräfte an der Spitze eines Hauses oder Unternehmens kennen das Gefühl nicht wertgeschätzt zu werden.

\section{Wertschätzung für die Geschäftsführung?}

In einem seit Jahren schwer defizitären Krankenhaus der Grund- und Regelversorgung wird ein neuer Geschäftsführer eingestellt. Seitens des kommunalen Krankenhausträgers besteht der wichtigste Auftrag des Geschäftsführers darin, die drohende Insolvenz abzuwenden, um so die Patientenversorgung in der Region und die Stellen der Mitarbeiter zu sichern. Mit Hilfe verschiedenster Maßnahmen zur Effizienzsteigerung und Erlösoptimierung zu denen auch ein Neubauprojekt gehört, gelingt es schließlich, das Haus innerhalb einiger Jahre aus den roten Zahlen zu führen. Die Mitarbeiterschaft, die lokale Presse und die politische Opposition fokussieren aber auf die Einsparungen von Personal und Versorgungsangebot. Der Geschäftsführer gilt trotz des wirtschaftlichen Erfolges und trotz der damit verbundenen Sicherung des generellen Versorgungsangebotes und der Arbeitsplätze als extrem unbeliebt. Auch wenn er sich dies 
nicht anmerken lässt, spürt er doch diese Ablehnung. Gefühle von Verbitterung stellen sich ein, er fühlt sich unfair und ungerecht bewertet.

Ethik-Projekte werden unter anderem wegen derartiger Phänomene ins Leben gerufen. Wie in dem oben beschriebenen Beispiel aus England (vgl. Abschnitt Staffordshire-Scandal, Kap. 1) birgt die Konzentration der Führungskräfte auf „Zahlen, Daten und Fakten“ die Gefahr, dass die Dimensionen der Kultur, der Kommunikation und der Ethik aus dem Blick verloren wird.

\section{Ethische Probleme aus einer Befragung von Führungskräften und Mitarbeitern eines Klinikums der Maximalversorgung}

- Wertschätzung: Mitarbeiter fühlen von Vorgesetzten nicht genügend anerkannt.

- Wertschätzung: Führungskräfte fühlen sich von Nachgeordneten nicht genügend anerkannt.

- Wertschätzung: Patient steht nicht wirklich im Zentrum!

- Patientenrechte und Menschenrechte in der Praxis oft vergessen!

- Wahrung der Intimsphäre ist zu achten

- Aufklärung defizitär

- „Ethik und Ökonomie ist Zentralthema.“

- Erhöhte Aggressivität von Diagnostik, Behandlung, Umgang und Sprache

- Wirtschaftlichkeitsdruck mindert Qualität der Versorgung

- „Sinnlose Medizin“- Maß der Therapie soll präziser bestimmt werden

- "Ich rette sterbende Patienten vor dem Haus“" (Palliativ)

- "Die Medizin folgt dem Geld - Ökonomisierung sehe ich mit Sorge.“

- DRG-0ptimierung bestimmt Diagnosen, Indikationen, Entlasszeitpunkt

- Ehrliche Dokumentation leidet

- Mikrobiologische Diagnostik nur für Private unbegrenzt, für andere limitiert (MVZ). Dadurch ggf. Verzögerungen in der Behandlung und ggf. mehr Fehler.

- Abrechnungsüberlegungen beeinflussen Indikationsstellung in Palliativmedizin: „Mehrleistung i.S. von Überschreitung der Pflegetage führt zu Rückzahlungszwang, d.h. erzielte Überschüsse gehen verloren. Palliativbetten müssen oft trotz Wartelisten freigehalten werden! Die Medizin muss dem Geldrahmen folgen- nicht umgekehrt.“

- „Palliativmedizin wird behindert durch Kassen und Controlling.“

- Fall X-Ambulanz: Erst die Finanzierung, dann erst die Behandlung! 


\subsection{Unternehmensleitbild und Menschenbild der Medizin}

Der originäre Auftrag von Krankenhäusern ist die gute medizinische Versorgung von Patienten. Alle Führungskräfte im Krankenhaus müssen letztendlich diesem zentralen Ziel, dem Sachziel, dienen. Dazu ist es erforderlich, „die Medizin“ zu kennen, ihre Entwicklungen zu verfolgen und dies nicht nur technisch und ökonomisch, sondern auch moralisch zu bewerten. Darüber hinaus kann ein Unternehmensleitbild ein Menschenbild beinhalten, das im medizinischen Handeln berücksichtigt werden soll.

Ein Krankenhaus, das medizinische Versorgung leistet, muss sich am Entwicklungsstand der Medizin orientieren. Es sollen die medizinischen Methoden und Verfahren zum Einsatz kommen, die in ihrer Wirksamkeit gut belegt sind. Leitlinien medizinischer Fachgesellschaften geben Standards vor, nach denen die Behandlung zu erfolgen hat. Eine fortwährende Weiterbildung der Ärzte muss diese Entwicklungen aufnehmen. Dies ergibt sich aus der Verpflichtung zur Wahl jener Verfahren, die dem Patienten voraussichtlich am besten helfen und am wenigsten schaden.

Auf den ersten Blick gibt es nur eine Medizin. Die Medizinstudierenden werden mit ihr trainiert, geprüft, in die Praxis entlassen. Wer jedoch über das Pflichtprogramm der Medizin hinausschaut weiß, dass es verschiedene Modelle des Menschen in der Medizin gibt, die mit unterschiedlichen Haltungen und Handlungen der Ärzte, Therapeuten und Pflegenden verbunden sind. Wenn neben der naturwissenschaftlich-technischen Seite auch eine anthropologische Dimension der Medizin akzeptiert wird, stellen sich Fragen nach der Beachtung der vollen Existenz eines Menschen im Prozess der medizinischen Handlungen und Entscheidungen.

Krankenhäuser können sich dafür entscheiden, ob ein bestimmtes Menschenbild für ihre Mitarbeiter leitend sein soll. Dies wäre eine der grundlegendsten ethischen Entscheidungen der Träger und der Führungskräfte.

Krankenhäuser können sich hinsichtlich eines „Stils“ und einer „Kultur“ von Medizin und Pflege unterscheiden. Dieser beruht neben dem Wertekanon mancher Häuser auch auf unterschiedlichen Denkmodellen, Paradigmen genannt, die für die Praxis wichtig sind. Im Kern geht es dabei um die Frage, ob und wieweit technische Arbeitsprozesse eingebettet sind in eine Versorgungskultur, in der die Person des Patienten gesehen wird oder nicht. „Personalisierte Medizin“, die heute von der molekularen und 
sozialepidemiologischen Forschung angestrebt wird, wird schon wesentlich länger von der „anthropologischen Medizin“ und von Teilen der „Psychosomatik" als Anspruch verfolgt. Hier geht es aber nicht primär um das menschliche Genom und Epigenom (das auch Einflüsse der Umwelt und des Verhaltens auf Gesundheit und Krankheit berücksichtigt), sondern die Person des Kranken mit ihrer Lebensgeschichte, ihren Zielen und Bedürfnissen soll im Mittelpunkt der Fürsorge stehen. Wenn in der Gesundheitspolitik und in zahllosen Klinikleitbildern davon die Rede ist, dass der Mensch im Mittelpunkt stehe, dann müsste das Haus mit allen in ihm Arbeitenden genau dafür sorgen. Wenn aber das Finanzierungssystem eine maximale Beschleunigung der Versorgungsprozesse belohnt und die Liegezeit der Patienten im Krankenhaus immer weiter reduziert wird, dann wird „Reparaturmedizin“ betrieben, zu Lasten einer echten personalisierten Medizin.

Was allgemein zu den Selbstverständlichkeiten einer menschenfreundlichen Medizin zählt wird heute für das Krankenhaus immer schwerer umzusetzen, weil Personal- und Zeitverknappung diese Form von Versorgung extrem erschweren. Umso größer ist die Herausforderung aller Verantwortlichen, sich diesem Trend zu widersetzen und sich für die Sicherstellung des Prinzips „Patient im Mittelpunkt“ einzusetzen.

„Aber folgendes dürfte unverzichtbar sein: Verständnis und Einfühlungsvermögen, Gesprächsbereitschaft und Geduld, die Fähigkeit zuzuhören und Mut zu machen sowie die Bereitschaft, seelische Probleme nicht bloßzu,somatisieren, indem Gefühle wie Angst und Hoffnungslosigkeit kurzerhand als Depression etikettiert werden, die nur mit Psychopharmaka zu behandeln sind." (Höffe 2002)

„Ohne Zuwendung ist alles nichts- Für eine Medizin der Zwischenmenschlichkeit. Zuwendung als Anerkennung des Patienten in seiner Unverwechselbarkeit. Medizin als Verbindung von Sachlichkeit und Zwischenmenschlichkeit." (Maio 2016)

Aktuelle Interviews mit Ärzten zeigen, dass für so viel Eingehen auf die Person des Patienten die Zeit und die Kontinuität der Zuständigkeiten nicht hinreichend gegeben sind. Die Ursache hierfür liegt im politisch bestimmten Finanzierungs- und Steuerungsmodell. Wenn die Aussage von T. v. Uexküll und T. Wesiack über die Humanmedizin zutrifft, dass „die Reduktion des Menschenbildes auf die mechanischen Zusammenhänge mit der Person des Patienten auch die Persönlichkeit des Arztes entwertet", dann handelt es sich um eine ethische Herausforderung. Es wird hier verständlich, dass die wachsende Außensteuerung der Medizin sowohl unter juristischen als auch unter gesundheitsökonomischen Kon- 
zepten von einer wachsenden Zahl von Ärzten, Therapeuten und Pflegenden als Demütigung, als Entwertung, als fehlende Wertschätzung erlebt wird. Dies dürfte ein Grund sein für die schwindende Attraktivität des Arbeitsplatzes Krankenhaus.

\section{Viktor von Weizsäcker und die anthropologische Medizin}

Die Tradition der „anthropologischen Medizin“ geht u.a. auf Viktor von Weizsäcker zurück. In seinen Texten geht es auch um die Ethik ärztlichen Denkens und Handelns. Dabei taucht der Begriff Ethik kaum auf. Diese ist quasi eingebettet in das hier entwickelte Menschenbild, das vom Arzt eine Verantwortlichkeit einfordert, die es zu einem unverzichtbaren Bestandteil seines Auftrages macht, den Patienten als Person einschließlich ihrer Biografie wahrzunehmen. Uexküll und Wesiack schreiben in diesem Sinne: „Ärztliche Ethik meint Verantwortung vor der irreversiblen Geschichte eines jeden menschlichen Lebens."

Die Anthropologische Medizin kämpft um die Beachtung des Subjektstatus der Patienten. Dieser wäre dann nicht einfach als „Träger" seiner Krankheit, er wäre auch als „Subjekt“ seiner Erkrankung zu sehen. Ein solches Verständnis prägt die „Form“ der Medizin: „Ich glaube, dass die klinische Medizin im Kampf um ihre Form steht." Es macht einen Unterschied, ob das ausführliche Gespräch zwischen Arzt und Patient vom Hause aus gefordert und gefördert wird. Dies prägt das „Klima“ eines Krankenhauses, seinen „Stil“ oder seine „Kultur“.

Kritisch äußert sich von Weizsäcker: „Im Krankensaal der Klinik gibt es ferner kein ordentliches Gespräch mit dem Kranken; die Kurve regiert die Stunde. Im Gespräch steckt aber das Subjekt, die Seele der Sache."

Das ethische Gebot zur Achtung der Autonomie des Patienten kann durchaus auch im Sinne v. Weizsäckers ausgelegt werden. Krankenhäuser, die Wert legen auf die Beachtung der Patientenrechte einschließlich des Rechts auf umfassende Information über Erkrankung und Therapie müssen genügend Zeit für Patientengespräche in ihre Prozessgestaltung einplanen. Und sie müssen darauf achten, dass die nötige Qualifikation für derlei Gespräche vorhanden ist. (v. Weizsäcker 1947)

Um der Verantwortung nicht nur der Ärzte, sondern aller Gesundheitsberufe gerecht zu werden muss dieses individuelle menschliche Leben wahrgenommen werden können. Dafür braucht es Zeit, Qualifikation, und organisatorische Unterstützung. In vielen Kliniken wird dies den einzelnen Personen überlassen, der Erfolgt ist abhängig von deren Persönlichkeit, Ressourcen und Engagement. Ziel einer patientenzentrierten Unternehmenskultur im Krankenhaus sollte es hingegen sein, die Verantwortung für eine „personalisierte Medizin und Pflege“ auch in die Hände der Organisation und damit aller Führungskräfte zu legen. Der freie Dialog zwischen den patientennahen Berufen und dem Management 
ist hier wichtig. Die Führungskräfte müssen wissen, was an der „Front der Versorgung“ faktisch geschieht und welche Probleme hier auftreten, um bei der Lösung derselben Unterstützung zu geben.

\section{Ethik-Projekt am Klinikum Nürnberg}

Das im Jahr 1999 gestartete „Ethik-Projekt am Klinikum Nürnberg“, einem der größten kommunalen Krankenhäuser Europas, verfolgte genau dieses Ziel einer Medizin, die der Person und dem Leben jedes einzelnen Patienten gerecht wird. Es ging auf die Initiative von Walter Gallmeiner zurück, damals Ärztlicher Vorstand und Chefarzt der Klinik für Hämatologie und Onkologie. Für Gallmeiner war es ein zentrales Anliegen, durch medizinische und pflegerische Ethik seine Vorstellung einer „Medizin nach Maß“ zu stärken, die zugleich eine „Beziehungsmedizin“ sein müsse. Zur heilkundlichen Aufgabe gehöre „... das Dabeisein, und zwar sowohl im Leben wie im Sterben“. Gallmeier betonte folgende Grundlagen seines Verständnisses von Heilkunde: „Naturwissenschaftliche Medizin, Realistische Medizin nach Maß, Beziehungsmedizin, anthropologische Medizin mit Blick in die Transzendenz."

Im Ethik- Projekt Nürnberg ging es uns auch um die Stärkung dessen, was als "Seele der Heilkunde“ bezeichnet wurde. Diese wurde durch die zunehmende Außensteuerung der Medizin als gefährdet angesehen. Es war wichtig, dass diese schwer beschreibbare „Seele“ im Krankenhaus nicht zerstört wurde durch Managementprozesse, die primär durch ökonomische Vorgaben geleitet werden.

Ein Krankenhaus sollte nicht den Eindruck einer Spaßveranstaltung oder eines Wellness-Centers erzeugen. Die Gründe, die Menschen als Patienten hierherführen, sind für sie sehr ernst. Die Kultur des Hauses sollte dies respektieren. Auch das gehört zum Thema Ethik.

\section{Das Krankenhaus als ein besonderer Ort}

In dem amerikanischen Roman „Begegnung am Abend“ von Brian Morton (1999) findet sich die Beschreibung einer Szene, in der ein alter Mann seinen Freund in einer New Yorker Klinik besucht. Die hier prosaisch beschriebene Empfindung beschreibt das Phänomen Krankenaus besser, als es viele analytische Texte können. Sinngemäß heißt es: Immer wenn er in eine Klinik kam, da merkte er, hier ist etwas Besonderes, hier wird gestorben, hier wird geboren. So eine Klinik ist nicht einfach eine Fabrik, das ist etwas Besonders. „Hier liegt was Heiliges“ sagte er, „schwer zu beschreiben."

Als Gegenbeispiel sei eine große Klinik in Norddeutschland erwähnt, die im Warteraum der Notaufnahme offenbar den Wartenden ein Unterhaltungsprogramm bieten will. Auch sterbenskranke Menschen, die hier manchmal sogar Stunden verbringen müssen, waren einem unter der Decke fest montierten 
Bildschirm ausgesetzt, der ohne Pause ein Video zeigt, in dem sich mittelalterliche Figuren und Monster auf alle möglichen Weisen gegenseitig bekämpften und grausam töteten. Dazu lief der Ton eines Radioprogramms mit Musik und Werbung. Es war nicht möglich, diesen Bildern und dem Lärm auszuweichen. Das Personal der ZNA verfügte über keine Möglichkeiten zum Stopp der Sendungen. Das Qualitätsmanagement bedankte sich für die eingegangenen Beschwerden. Drei Wochen später lief dasselbe Programm immer noch.

Für kirchliche Krankenhäuser ist die Achtung des Subjekts ein Aspekt ihrer Profilierung. Kritisch fragt eine vom Katholischen Krankenhausverband und der Caritas geförderte Studie, „ob der Mensch als Subjekt und leidende Person in der nach ökonomischen Gesichtspunkten umstrukturierten Medizin überhaupt noch eine vorrangige Rolle spielt." Das Ergebnis ist nicht überraschend: „Die nach ökonomischen Gesichtspunkten durchgeführten Rationalisierungsmaßnahmen in der Medizin lassen für solche zeitaufwändige, den Patienten in seiner Subjektivität wahrnehmende Kommunikation immer weniger Zeit und Kraft." Die Studie schließt mit dem Hinweis, dass für kirchliche Krankenhäuser die „Wahrnehmung des Patienten als Subjekt (...) ein Wettbewerbsvorteil sein“ kann.

\section{Gesundheit als Potenzial}

Neben dem „Menschenbild“ in der praktizierten Medizin hat auch der „Gesundheitsbegriff" eine ethische Relevanz. Betrachtet man die Gesundheit als einen Zustand und den Menschen als ein Werkstück, das im Sinne eines Objekts umfassend bearbeitet werden kann, so ist der Patient wenig als Person beteiligt. Dann sollen Ärzte diesen Zustand möglichst umfassend zum Ziel ihrer Arbeit machen. Wird hingegen Gesundheit als Potenzial betrachtet, das sich aus biogenetischen und lebensgeschichtlich erworbenen Aspekten zusammensetzt, dann wird Gesundheitsförderung zu einem wichtigen Ziel der Medizin. Letztere wird zu einem Thema der Gestaltung kollektiver Lebensbedingungen und der individuellen Lebensführung des lebendigen Patienten. Aufgabe des Klinikpersonals wäre die Förderung dieses Potenzials. Ein wichtiges Medium ist das Gespräch. (Bircher u. Wehkamp 2006)

Es gibt durchaus Krankenhäuser in Deutschland, die seitens ihrer Führung und Organisation auf die Wahl grundlegender Paradigmen achten. Reha-Kliniken und Psychosomatische Kliniken sind hier besonders zu nennen. Ihr Konzept gibt der Arbeit mit den Patienten eine hohe Bedeutung. Der Achtung und Wertschätzung von Menschen wird jenseits der puren Behandlung ein hoher Stellenwert eingeräumt. Die ethische Herausforderung, in einer durch Technik und Ökonomisierung geprägten 
Medizin den Bedürfnissen der Menschen nach Anerkennung, Empathie und Wärme gerecht zu werden wird auf diese Weise angenommen.

\subsection{Ethische Konflikte im Rahmen von Diagnostik und Therapie}

Entscheidungen über medizinische Maßnahmen werden von Ärzten und Pflegenden getroffen, wobei letztere häufig darüber entscheiden, wann ein Alarm ausgelöst wird und wann welche Ärzte gerufen werden. Alle diese Entscheidungen enthalten die Möglichkeit dem Patienten zu helfen oder ihm zu schaden. Dies gilt für jede Maßnahme und für jede Unterlassung. Deshalb müssen die Entscheider damit leben, dass sie sich durch ihre Entscheidungen schuldig machen können, gegenüber anderen und vor allem gegenüber sich selbst. Viele Entscheidungen sind nicht nur durch die aktuelle Situation des Patienten bestimmt, sondern auch durch die Erfahrung, Qualifikation und Persönlichkeit des Arztes und durch die Zusammensetzung der umgebenden Teams. Potentiell gibt es auch vielerorts eine Einflussnahme durch wirtschaftliche Anreize beziehungsweise die Verfügbarkeit von Ressourcen. Um gute Entscheidungen treffen zu können, ist deshalb die Fähigkeit zur Reflexion der zugrundeliegenden Kriterien von besonderer Bedeutung.

Wenn es gelingt, die Entscheidungsprozesse und Kriterien transparent zu machen und mit anderen Personen des Behandlungsteams zu besprechen, dann kann dies dem Patienten, der Teamkultur und dem Gewissen des Arztes und seiner Teammitglieder nützen. In klinischen Ethik-Projekten geraten häufig Situationen in das Blickfeld, in denen bösartige, chronifizierte Konflikte zwischen Mitarbeitern, Abteilungen und Berufsgruppen die Arbeit ganzer Stationen und darüber hinaus lähmen. Neben unangenehmen Auseinandersetzungen mit schlechter Ausstrahlung sind Krankmeldungen und eine hohe Personalfluktuation die Folge. Insgesamt leidet sowohl die Effektivität als auch die Effizienz. Ethik-Teams und Führungskräfte mit Ethik-Kompetenz können in solchen Fällen wertvolle Mediationen leisten. Gegenüber rein psychologischer Supervision besteht hier der Vorteil, dass die Probleme nicht als innerpsychische interpretiert werden, was beim Personal durch die damit verbundene Stigmatisierung oft Abwehrreaktionen hervorruft.

Besonders häufig finden sich Konflikte zwischen Ärzten und Pflegenden über Maß und Intensität von Maßnahmen, vor allem wenn es um Reanimationen, Intensivmedizin, Chemotherapie und eingreifende chirurgische Verfahren geht. Pflegende berichten in Ethik-Projekten häufig von Gewissenskonflikten, wenn sie helfend zu ärztlichen Handlungen hinzugezogen werden, die sie selbst für nicht angemessen halten. Störungen 
der Zusammenarbeit entstehen, wenn Entscheidungsbegründungen nicht transparent sind. Ärzte klagen häufig über Pflegende, die ihre Arbeit sabotieren indem sie sie „auflaufen lassen. “Ethikberatung, Fallbesprechungen und Möglichkeiten der Aussprache in Ethik-Cafés können für Klärungen sorgen, die das Arbeitsklima und letztlich die Patientenversorgung positiv beeinflussen.

Oft sind es ganze Kliniken, Institute oder andere Abteilungen des Krankenhauses die sich in Ethik-Projekten einbringen, um sich Hilfe bei der Lösung chronischer Konflikte zu holen. Führungskräfte in ihrer Verantwortung für die Effizienz und Effektivität der Abläufe und für das Personalmanagement sollten von solchen Ressourcen Gebrauch machen.

\subsection{Grundkonflikt zwischen Medizinethik und betriebswirtschaftlichen Unternehmenszielen}

Krankenhäuser, in Europa einst als konfessionelle Pflegestätten und Orte christlicher Barmherzigkeit eingerichtet, wurden im 19. Jahrhundert Orte der Medizin. Bis heute wurden sie zunehmend in staatliche Rechtsordnungen und politisch gestaltete Gesundheitssysteme eingebettet. In Deutschland wurde schrittweise nahezu die gesamte Bevölkerung mit dem Anspruch auf eine dem Stand der medizinischen Wissenschaft gemäße Versorgung ausgestattet. Krankenhäuser haben diesen Auftrag mit zu erfüllen. Für die Bevölkerung wird dabei nicht nur der technische Aspekt der Medizin bereitgestellt, sondern auch ihre sozialpolitischen und ethischen Grundsätze. Unbestritten sollen die Kliniken dem Wohl des Patienten dienen und der Bevölkerung einen Ort der Daseinsfürsorge bieten. Dabei wurde die Entwicklungs- und Steuerungshoheit für die Medizin bis vor wenigen Jahren den Ärzten überlassen.

Diese Hoheit wurde im neuen Jahrtausend schrittweise in die Hände betriebswirtschaftlicher Fachkräfte gelegt. Die Binnensteuerung der Medizin wurde - nicht nur in Deutschland - schrittweise durch eine mehr oder weniger gesundheitsökonomische Steuerung ersetzt. Die Chefärzte und Pflegedirektoren wurden kaufmännischen Vorständen bzw. Geschäftsführern unterstellt. Diese wurden mit dem Auftrag versehen, Unwirtschaftlichkeit zu beseitigen, die Effizienz und Effektivität der Versorgungsprozesse zu steigern und letztendlich die Kostenentwicklung in Schranken zu weisen. Mit den privatwirtschaftlich aufgestellten Krankenhauskonzernen wurde dann auch das Ziel der Erwirtschaftung von Renditen verbindlich.

Was den leitenden Ärzten nicht gelungen war, sollten nun „ökonomische“ Führungskräfte leisten. Dazu mussten aus den Krankenanstalten Gesund- 
heitsunternehmen werden, die Verwaltungen mussten sich in vorausschauendes Management verwandeln und das Gesundheitssystem wurde tendenziell in einen Gesundheitsmarkt umgewandelt. Seitdem Krankenhäuser dem Wettbewerb ausgesetzt sind und insolvent werden können ist eine betriebswirtschaftlich erfolgreiche Führung die Voraussetzung ihrer Zukunftssicherung geworden. Der Erhalt der wirtschaftlichen Grundlagen und darin eingeschlossen die Erwirtschaftung von Gewinnen zur Sicherung notwendiger Investitionen ist aus sozialethischer Sicht ein ebenso wertvolles Ziel wie die Ermöglichung eines hochwertigen, aber zugleich für die Bevölkerung finanzierbaren Gesundheitssystems.

Krankenhäuser haben somit einen doppelten Auftrag. Zum einen sollen sie hochwertige Medizin für alle Menschen bereitstellen, die dieser bedürfen. Zum anderen sollen sie Kernbestandteil eines politisch gestalteten Gesundheitssystems sein, das an den Interessen der ganzen Bevölkerung orientiert ist. Das Gesundheitssystem wiederum wurde zunehmend in die Hände privatwirtschaftlicher Anbieter gegeben, so dass neben einer Gewinn- auch eine Profitorientierung zu den Zielen vieler Krankenhäuser hinzugefügt wurde. Fortan sind alle Mitarbeiter, besonders aber alle Führungskräfte des Krankenhauses auf medizinische und medizinethische Ziele verpflichtet, zugleich aber auch auf betriebswirtschaftliche. Damit ist strukturell ein Ziel- und Wertekonflikt Bestandteil der alltäglichen Führungsarbeit, und es zeigt sich, dass ein sehr großer Teil der Kliniken dieser Herausforderung nur sehr unzureichend gewachsen ist (s. Abb. 3).

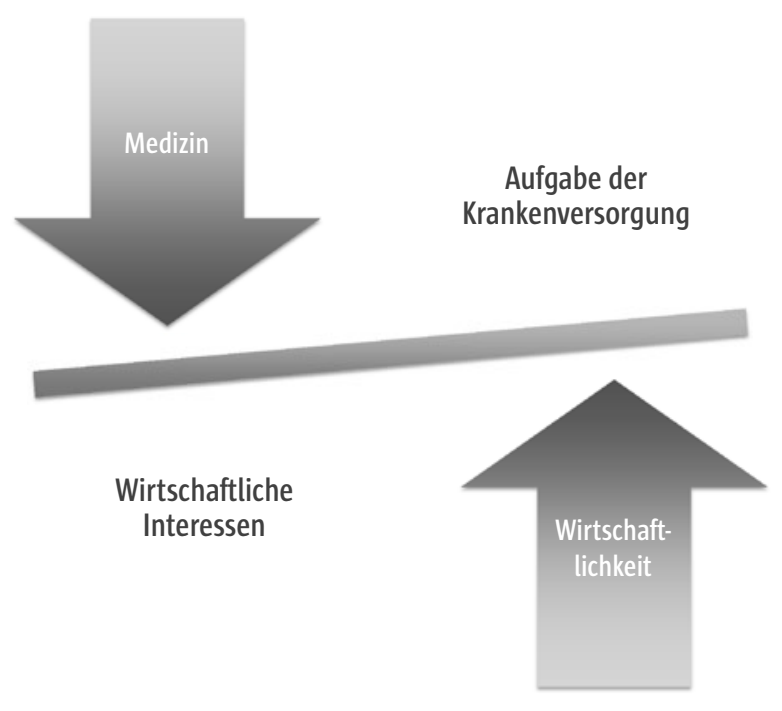


Beide Seiten - die der Geschäftsführung und die medizinische - wissen sehr häufig nicht, wie ihre jeweiligen Ziele konkret umgesetzt werden können, ohne sich gegenseitig ins Gehege zu kommen. Wie können die Ziele „wirtschaftliche Existenzsicherung des Unternehmens “ und „bestmögliche Versorgung von Patienten“ optimal balanciert werden? Die Lösung der Aufgabe wird leichter indem man sich klarmacht, dass sich an dieser das Management fordernden Schnittstelle auch um eine Schnittstelle zweier grundlegender unterschiedlicher ethischer Denkmodelle handelt: Prinzipienethik (Deontologie) versus Nutzenethik (Utilitarismus) (s. Kap. 2). Die „hippokratische“ Medizin verlangt die strikte Befolgung ihrer Grundsätze, vor allen zum individuellen Patientenwohl einschließlich der Schadensvermeidung. Unternehmensführungen sollen diese Ziele zwar auch für das gesamte Klinikum übernehmen, werden aber zuerst an wirtschaftlichen Erfolgszahlen gemessen. Die Finanzierungsordnung im Gesundheitswesen und in der Gesundheitswirtschaft erzwingt die Dominanz betriebswirtschaftlicher Ziele, die gleichwohl auf rechtlicher Seite nicht zu Lasten des Patientenwohls gehen sollen.

Faktisch besteht in Deutschland zwischen dem Wohl des Patienten und dem Wohl des Krankenhausunternehmens keine grundsätzliche Harmonie. Konflikte um knappe Mittel, Personalengpässe, Investitionsstaus führen auf Seiten der Patientenversorgung immer wieder zu Mangelsituationen, verdeckten Rationierungen (zum Beispiel von Zeit) und Konfrontationen zwischen Ärzten, Therapeuten und Pflegenden auf der einen und dem kaufmännischen Management auf der anderen Seite. Auf der anderen Seite besteht die Gefahr der angebotsinduzierten Überversorgung, wenn Mengenausweitungen aus betriebswirtschaftlichen Gründen angestrebt werden. Der Kern dieser Konflikte ist ethischer Natur, der sich mit der wissenschaftlichen Gesundheitsökonomie und der herkömmlichen Betriebswirtschaft niemals allein lösen lässt. Es braucht ethisches Wissen und Ethik-Kompetenz um in den unvermeidbaren Situationen des Dilemmas entscheidungsfähig zu bleiben. Die Zwickmühlen, in denen sich die Krankenhäuser befinden, lassen sich weder rein medizinethisch noch rein ökonomisch auflösen. Kompromisse müssen gefunden und der Mitarbeiterschaft überzeugend vermittelt werden. Vor allem braucht es belastbare Wege des wechselseitigen Informationsaustauschs, der Transparenz und eine Kultur des wechselseitigen Respekts.

\section{Ethische Konflikte zwischen Medizin und Betriebswirtschaft}

- Dürfen wirtschaftliche Ziele des Hauses die Diagnostik-und Therapieentscheidungen beeinflussen? 
- Wie sind Patienteninteresse und Unternehmensinteressen konkret zu balancieren?

- Was darf eine Behandlung kosten? Wie viel Aufwand ist angemessen?

- Was tun, wenn eine fachgerechte medizinische Behandlung dem Krankenhaus Defizite beschert?

- Wie viel Geld aus den Gesamterlösen soll für das Personal und wie viel für Investitionen (z.B. in Medizintechnologie) ausgegeben werden?

- Ist es akzeptabel, einem bedürftigen Patienten Behandlungen vorzuenthalten, um das Gesamtsystem bezahlbar zu halten?

- Wie sind knappe Mittel innerhalb des Unternehmens zu verteilen? Nach welchen Kriterien?

Die Möglichkeiten des Managements zur Lösung solcher konfliktträchtigen Fragen sollten allerdings nicht überschätzt werden. Das gesamte Problemfeld „Medizinethik- Betriebswirtschaft- Volkswirtschaft-Gesellschaft" muss sich diesen Fragen stellen. Mit der Medizinethik allein wird man nicht zurechtkommen. Auch in Wirtschaft Politik und Öffentlichkeit muss die Frage gestellt werden, wie viel die Gesundheit der Menschen der Gemeinschaft „wert“ sein soll, was in der Verantwortung des Einzelnen oder der Gesellschaft liegt, welche Ziele die Medizin verfolgen soll, wie alt wir werden wollen, sollen oder gar dürfen- um welchen Preis und auf wessen Kosten.

\section{Empfehlungen auf Basis eines Forschungsprojekts zur „Ökonomisierung patientenbezogener Entscheidungen im Krankenhaus“}

Es muss damit gerechnet werden, dass die Mittel - gemessen an der zunehmenden Inanspruchnahme der Krankenhäuser - weiterhin knapp bleiben. Deshalb wird es darauf ankommen, die medizinische und die wirtschaftliche Steuerung des Krankenhauses so zu gestalten, dass Rahmenbedingungen geschaffen werden, die es dem Arzt erlauben, patientenbezogene Entscheidungen zu fällen, die einerseits der Begrenztheit der Ressourcen Rechnung tragen und zum anderen ethisch vertretbar sind.

Allein auf der Ebene des Managements lassen sich die geschilderten Probleme jedoch nicht lösen. Aus unserer Sicht muss die Investitions-Finanzierung neu ausgerichtet werden, Überkapazitäten sollten beseitigt bzw. vermieden werden, die Grund- und Regelversorgung sollte neu definiert werden und die DRG- Bewertungsrelationen sollten an die Kostenstrukturen besser angepasst werden. Die Notfallambulanzen und die Extremkostenfälle sollten kostendeckend finanziert werden, die Dokumentation und Qualitätssicherung sollten vereinfacht und transparenter werden. (Naegler u. Wehkamp 2016) 


\section{Weiterführende Literatur}

Deichert U, Höppner W, Steller I (Hrsg) (2016) Traumjob oder Albtraum? Chefarzt w/m. Springer Berlin, Heidelberg

Lohfert C (2013) Das medizinische Prinzip: Handbuch für das Krankenhaus der Zukunft. Albrecht Knaus Verlag München

Marckmann G (2015) Praxisbuch Ethik in der Medizin. Medizinisch Wissenschaftliche Verlagsgesellschaft Berlin Pramstaller PP (2016) Rettet die Medizin. Medizinisch Wissenschaftliche Verlagsgesellschaft Berlin

Unschuld PU (2014) Ware Gesundheit: Das Ende der klassischen Medizin, 3. Auflage. C.H. Beck München

\section{Zitierte literatur}

Bircher J, Wehkamp KH, (2006) Das ungenutzte Potenzial der Medizin. Rüffer und Rub Zürich Höffe 0 (2002) Medizin ohne Ethik? Suhrkamp Frankfurt/Main Luhmann N (1989) Gesellschaftsstruktur und Semantik. 361, 371. Suhrkamp Frankfurt/Main Maio G (2016) Ohne Zuwendung ist alles nichts - Für eine Medizin der Zwischenmenschlichkeit. In: Deichert U, Höppner W, Steller I (Hrsg) Traumhob oder Albtraum - Chefarzt m/w. Springer Berlin Heidelberg

Naegler H, Wehkamp K-H, Auszug aus einem Vortrag im Rahmen der Berliner Wirtschaftsgespräche 16.11.2016 von Weizsäcker V (1947) Klinische Studien Stuttgart 